Stud. Univ. Babeş-Bolyai Math. 63(2018), No. 1, 141-151

DOI: 10.24193/subbmath.2018.1.09

\title{
Quintic B-spline method for numerical solution of fourth order singular perturbation boundary value problems
}

\author{
Ram Kishun Lodhi and Hradyesh Kumar Mishra
}

\begin{abstract}
In this communication, we have studied an efficient numerical approach based on uniform mesh for the numerical solutions of fourth order singular perturbation boundary value problems. Such type of problems arises in various fields of science and engineering, like electrical network and vibration problems with large Peclet numbers, Navier-Stokes flows with large Reynolds numbers in the theory of hydrodynamics stability, reaction-diffusion process, quantum mechanics and optimal control theory etc. In the present study, a quintic B-spline method has been discussed for the approximate solution of the fourth order singular perturbation boundary value problems. The convergence analysis is also carried out and the method is shown to have convergence of second order. The performance of present method is shown through some numerical tests. The numerical results are compared with other existing method available in the literature.
\end{abstract}

Mathematics Subject Classification (2010): 65L10.

Keywords: Fourth order singular perturbation boundary value problem, quintic B-spline, quasilinearization, uniform mesh, convergence analysis.

\section{Introduction}

We consider the fourth order singular perturbation boundary value problem

$$
\begin{gathered}
-\varepsilon y^{i v}(t)-p(t) y^{\prime \prime \prime}(t)+q(t) y^{\prime \prime}(t)+r(t) y(t)=f(t), \quad t \in[a, b], \\
y(a)=\eta_{1}, \quad y(b)=\eta_{2}, \quad y^{\prime \prime}(a)=\eta_{3}, \quad y^{\prime \prime}(b)=\eta_{4} .
\end{gathered}
$$

where $\eta_{1}, \eta_{2}, \eta_{3}$ and $\eta_{4}$ are finite real constants and $\varepsilon$ is a small positive parameter, such that $0<\varepsilon \ll 1$. Moreover, we assume that the functions $p(t), q(t), r(t)$ and $f(t)$ are sufficiently smooth. Further, the problem (1.1) is called non-turning point problem if $p(t) \geq \alpha>0$ throughout the interval $[a, b]$, where $\alpha$ is some positive constant and boundary layer will be in the neighbourhood of $t=a$ [9]. In the same 
vein, if the $p(t)$ vanishes at $t=0$, then it becomes a turning point problem. In that scenario, the boundary layer will be at both the end points $t=a$ and $t=b[2]$.

Singular perturbation problems are engendered by multiplication of a small positive parameter $\varepsilon$ to highest derivative term of differential equation with boundary conditions. Many scholars have studied the analytical and numerical solutions of these problems, but sometimes they found that the classical numerical methods failed to get good approximate solutions of singular perturbation problems. That's why they have gone for the non classical methods. In the last few decades, many researchers have discussed the numerical solutions of singular perturbation problems. Most of the researchers have studied the numerical solutions of second order singular perturbation problems $[5,10,11,12,13,17,19,20,21,22,29]$. Only a few researchers have focused the numerical solutions of higher order singular perturbation problems $[3,24,23,28,27]$. Lodhi and Mishra $[14,15]$ have suggested the computational technique for numerical solutions of fourth order singular singularly perturbed and self adjoint boundary value problems. Raja and Tamilselvan [23] have designed a shooting method on a Shishkin mesh to solve reaction-diffusion type problems. Mishra and Saini [18] have used initial value technique for the numerical solution of fourth order singularly perturbed boundary value problems. Sarakhsi et al [25] have studied the existence of boundary layer problem. Parameter uniform numerical scheme to solve fourth order singularly perturbed turning points problems have been presented by Geetha and Tamilselvan [7]. Sharma et al. [26] have done the survey on singularly perturbed turning point and interior layers problem. Geetha et al. [8] have applied parameter uniform numerical method based on Shishkin mesh for third order singularly perturbed turning point problems exhibiting boundary layers.

This paper describes a quintic B-spline approach for the numerical solution of fourth order singular perturbation boundary value problems and it has been proved to be second order convergence. The paper is organized as follows: In section 2, we describe the quintic B-spline method. Convergence analysis is established in section 3. Quasilinearization method is discussed in section 4. Section 5 gives the numerical results which substantiate the theoretical aspects. Finally, we discuss the conclusions in section 6 .

\section{Quintic B-spline Method}

We divide the interval $[a, b]$ into $N$ equal subinterval and we choose piecewise uniform mesh points represented by $\pi=\left\{t_{0}, t_{1}, t_{2}, \ldots, t_{N}\right\}$, such that $t_{0}=a, t_{N}=b$ and $h=\frac{b-a}{N}$ is the piecewise uniform spacing. We define $L_{2}[a, b]$ as a vector space of all the integrable functions on $[a, b]$, and $X$ be the linear subspace of $L_{2}[a, b]$. Now we define 


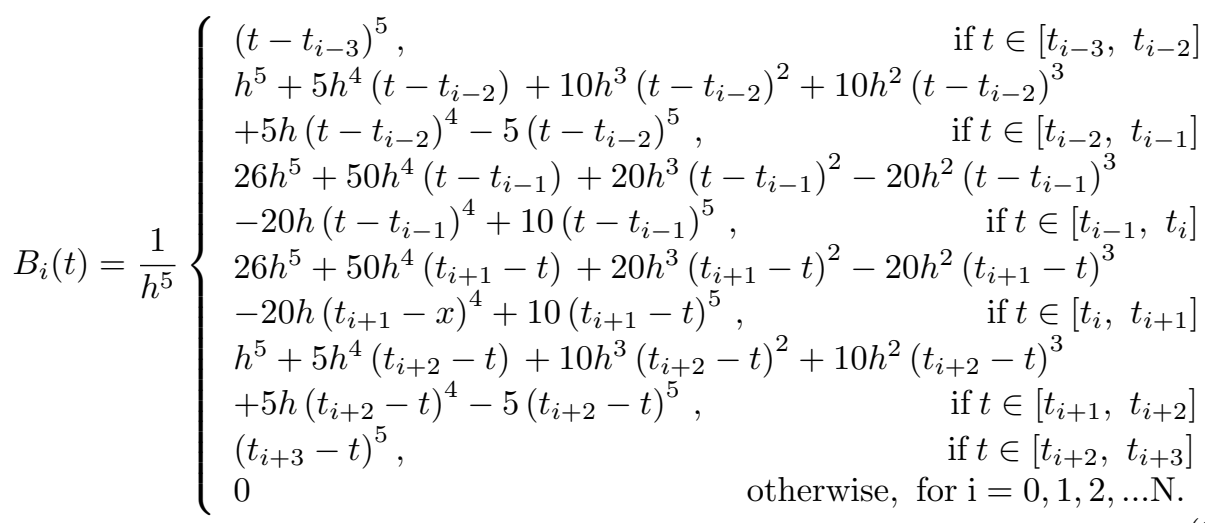

We introduce six additional knots as $t_{-3}<t_{-2}<t_{-1}<t_{0}$ and $t_{N+3}>t_{N+2}>$ $t_{N+1}>t_{N}$. From equation (2.1), we can easily check that each of the functions $B_{i}(t)$ is four times continuously differentiable on the entire real line. Also, the values of $B_{i}(t), B_{i}^{\prime}(t), B_{i}^{\prime \prime}(t), B_{i}^{\prime \prime \prime}(t)$ and $B_{i}^{i v}(t)$ at the nodal points are given in Table 1.

Table 1. Quintic B-spline basis and its derivative function values at nodal points

\begin{tabular}{llllllll}
\hline$B(t)$ & $t_{i-3}$ & $t_{i-2}$ & $t_{i-1}$ & $t$ & $t_{i+1}$ & $t_{i+2}$ & $t_{i+3}$ \\
\hline$B_{i}(t)$ & 0 & 1 & 26 & 66 & 26 & 1 & 0 \\
$B_{i}^{\prime}(t)$ & 0 & $5 / h$ & $50 / h$ & 0 & $-50 / h$ & $-5 / h$ & 0 \\
$B_{i}^{\prime \prime}(t)$ & 0 & $20 / h^{2}$ & $40 / h^{2}$ & $-120 / h^{2}$ & $40 / h^{2}$ & $20 / h^{2}$ & 0 \\
$B_{i}^{\prime \prime \prime}(t)$ & 0 & $60 / h^{3}$ & $-120 / h^{3}$ & 0 & $120 / h^{3}$ & $-60 / h^{3}$ & 0 \\
$B_{i}^{i v}(t)$ & 0 & $120 / h^{4}$ & $-480 / h^{4}$ & $720 / h^{4}$ & $-480 / h^{4}$ & $120 / h^{4}$ & 0 \\
\hline
\end{tabular}

Let $\Omega=\left\{B_{-2}, B_{-1}, B_{0}, B_{1}, \ldots \ldots, B_{N-1}, B_{N}, B_{N+1}, B_{N+2}\right\}$ and let $\phi_{5}(\pi)=$ $\operatorname{span} \Omega$. The function $\Omega$ is linearly independent on $[a, b]$, thus $\phi_{5}(\pi)$ is $(N+5)$ dimensional. Even one can show that $\phi_{5}(\pi) \subseteq$ subspace $X$. Let $L$ be a linear operator whose domain is $X$ and whose range is also in $X$. Now we define

$$
S(t)=\sum_{i=-2}^{N+2} c_{i} B_{i}(t),
$$

be the approximate solution of the problem (1.1) with boundary conditions (1.2), where $c_{i}^{\prime} s$ is an unknown coefficient and $B_{i}(t)^{\prime} s$ a fifth degree spline function. To solve fourth order singularly perturbed two point boundary value problems, the spline functions are evaluated at nodal points $t=t_{i}(i=0,1,2, \ldots, N)$ which are needed for the solution.

From Table 1 and equation (2.2), we obtain the following relationships:

$$
\begin{gathered}
y\left(t_{i}\right)=S\left(t_{i}\right)=c_{i-2}+26 c_{i-1}+66 c_{i}+26 c_{i+1}+c_{i+2} \\
m\left(t_{i}\right)=S^{\prime}\left(t_{i}\right)=\frac{1}{h}\left(-5 c_{i-2}-50 c_{i-1}+50 c_{i+1}+5 c_{i+2}\right)
\end{gathered}
$$




$$
\begin{gathered}
M_{i}=S^{\prime \prime}\left(t_{i}\right)=\frac{1}{h^{2}}\left(20 c_{i-2}+40 c_{i-1}-120 c_{i}+40 c_{i+1}+20 c_{i+2}\right) \\
T_{i}=S^{\prime \prime \prime}\left(t_{i}\right)=\frac{1}{h^{3}}\left(-60 c_{i-2}+120 c_{i-1}-120 c_{i+1}+60 c_{i+2}\right) \\
F_{i}=S^{i v}\left(t_{i}\right)=\frac{1}{h^{4}}\left(120 c_{i-2}-480 c_{i-1}+720 c_{i}-480 c_{i+1}+120 c_{i+2}\right)
\end{gathered}
$$

Moreover, $m_{i}, M_{i}, T_{i}$ and $F_{i}$ can be used to approximate values of $y^{\prime}\left(t_{i}\right), y^{\prime \prime}\left(t_{i}\right)$, $y^{\prime \prime \prime}\left(t_{i}\right)$ and $y^{i v}\left(t_{i}\right)$.

Since $S(t)$ is an approximate solution, it will satisfy equation (1.1) with boundary conditions (1.2). Hence we get

$$
-\varepsilon S^{i v}(t)-p(t) S^{\prime \prime \prime}(t)+q(t) S^{\prime \prime}(t)+r(t) S(t)=f(t),
$$

and

$$
S(a)=\eta_{1}, \quad S(b)=\eta_{2}, \quad S^{\prime \prime}(a)=\eta_{3}, \quad S^{\prime \prime}(b)=\eta_{4}
$$

Discretizing equation (2.8) at the nodal points $t_{i}(i=0,1, \ldots, N)$, we have

$$
-\varepsilon S^{i v}\left(t_{i}\right)-p\left(t_{i}\right) S^{\prime \prime \prime}\left(t_{i}\right)+q\left(t_{i}\right) S^{\prime \prime}\left(t_{i}\right)+r\left(t_{i}\right) S\left(t_{i}\right)=f\left(t_{i}\right),
$$

Using equations (2.3)-(2.7) in above equation and simplifying, we obtain

$$
\begin{aligned}
& -\frac{\varepsilon}{h^{4}}\left\{120 c_{i-2}-480 c_{i-1}+720 c_{i}-480 c_{i+1}+120 c_{i+2}\right\} \\
& -\frac{p_{i}}{h^{3}}\left\{-60 c_{i-2}+120 c_{i-1}-120 c_{i+1}+60 c_{i+2}\right\} \\
& +\frac{q_{i}}{h^{2}}\left\{20 c_{i-2}+40 c_{i-1}-120 c_{i}+40 c_{i+1}+20 c_{i+2}\right\} \\
& +r_{i}\left\{c_{i-2}+26 c_{i-1}+66 c_{i}+26 c_{i+1}+c_{i+2}\right\}=f_{i} h^{4},
\end{aligned}
$$

where $p_{i}=p\left(t_{i}\right), q_{i}=q\left(t_{i}\right), r_{i}=r\left(t_{i}\right)$ and $f_{i}=f\left(t_{i}\right)$. After simplifying above equation, we get

$$
\gamma_{1}\left(t_{i}\right) c_{i-2}+\gamma_{2}\left(t_{i}\right) c_{i-1}+\gamma_{3}\left(t_{i}\right) c_{i}+\gamma_{4}\left(t_{i}\right) c_{i+1}+\gamma_{5}\left(t_{i}\right) c_{i+2}=f_{i} h^{4},
$$

where

$$
\begin{aligned}
& \gamma_{1}\left(t_{i}\right)=-120 \varepsilon+60 p_{i} h+20 q_{i} h^{2}+r_{i} h^{4}, \\
& \gamma_{2}\left(t_{i}\right)=480 \varepsilon-120 p_{i} h+40 q_{i} h^{2}+26 r_{i} h^{4}, \\
& \gamma_{3}\left(t_{i}\right)=-720 \varepsilon-120 q_{i} h^{2}+66 r_{i} h^{4}, \\
& \gamma_{4}\left(t_{i}\right)=480 \varepsilon+120 p_{i} h+40 q_{i} h^{2}+26 r_{i} h^{4}, \\
& \gamma_{5}\left(t_{i}\right)=-120 \varepsilon-60 p_{i} h+20 q_{i} h^{2}+r_{i} h^{4}, \quad \text { for } i=0,1, \ldots, N .
\end{aligned}
$$

From the boundary conditions, we get the following equations

$$
\begin{gathered}
c_{-2}+26 c_{-1}+66 c_{0}+26 c_{1}+c_{2}=\eta_{1}, \\
c_{N-2}+26 c_{N-1}+66 c_{N}+26 c_{N+1}+c_{N+2}=\eta_{2}, \\
20 c_{-2}+40 c_{-1}-120 c_{0}+40 c_{1}+20 c_{2}=\eta_{3} h^{2},
\end{gathered}
$$

and

$$
20 c_{N-2}+40 c_{N-1}-120 c_{N}+40 c_{N+1}+20 c_{N+2}=\eta_{4} h^{2} .
$$

Coupling equations (2.11)-(2.15) lead to a system of $(N+5)$ linear equations $A Y=D$ in the $(N+5)$ unknowns, where

$$
\begin{gathered}
Y=\left[c_{-2}, c_{-1}, c_{0}, c_{1}, \ldots, c_{N-1}, c_{N}, c_{N+1}, c_{N+2}\right]^{T} \\
D=\left[\eta_{1}, \eta_{3} h^{2}, f_{0} h^{4}, f_{1} h^{4}, \ldots, f_{N-1} h^{4}, f_{N} h^{4}, \eta_{4} h^{2}, \eta_{2}\right]^{T}
\end{gathered}
$$


and the coefficient matrix $A$ is given by

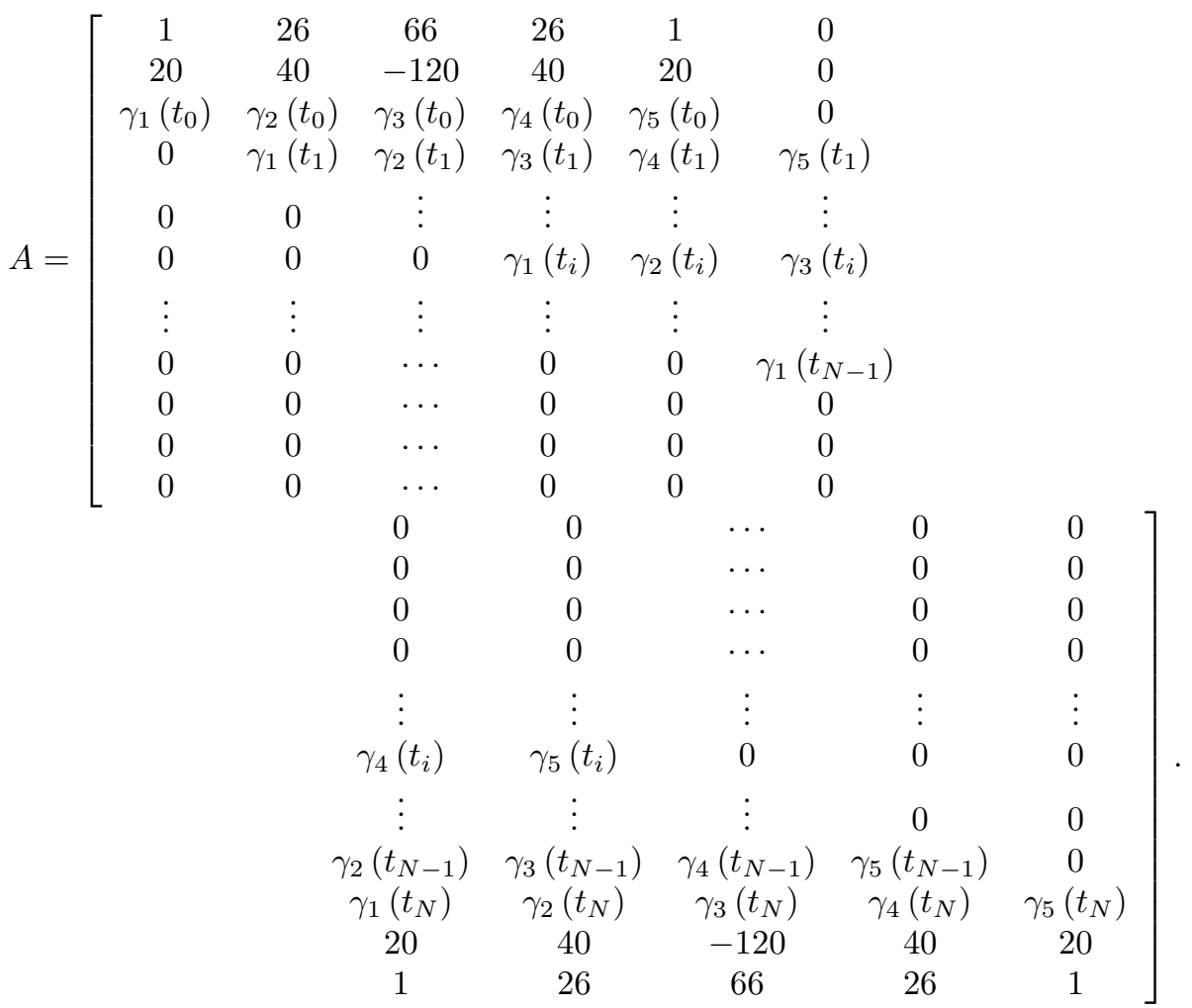

Since $A$ is a non-singular matrix, so we can solve the system $A Y=D$ for $c_{-2}, c_{-1}, c_{0}, c_{1}, c_{2}, \ldots, c_{N-2}, c_{N-1}, c_{N}, c_{N+1}, c_{N+2}$ substituting these values into equation (2.2), we get the required approximate solution.

\section{Derivation for convergence}

In this section, a technique is portrayed which will ascertain the truncation error for the quintic B-spline method over the whole range $a \leq t \leq b$. Here, we suppose that function $y(t)$ has continuous derivatives in the whole range.

We calculate the following relationships by comparing the coefficients of $c_{i}(i=-2,-1,0,1, \ldots, N, N+1, N+2)$. From equations (2.3)-(2.7), we have

$$
\begin{array}{r}
S^{\prime}\left(t_{i-2}\right)+26 S^{\prime}\left(t_{i-1}\right)+66 S^{\prime}\left(t_{i}\right)+26 S^{\prime}\left(t_{i+1}\right)+S^{\prime}\left(t_{i+2}\right) \\
=\frac{1}{h}\left\{-5 y\left(t_{i-2}\right)-50 y\left(t_{i-1}\right)+50 y\left(t_{i+1}\right)+5 y\left(i_{i+2}\right)\right\} \\
S^{\prime \prime}\left(t_{i-2}\right)+26 S^{\prime \prime}\left(t_{i-1}\right)+66 S^{\prime \prime}\left(t_{i}\right)+26 S^{\prime \prime}\left(t_{i+1}\right)+S^{\prime \prime}\left(t_{i+2}\right) \\
=\frac{1}{h^{2}}\left\{20 y\left(t_{i-2}\right)+40 y\left(t_{i-1}\right)-120 y\left(t_{i}\right)+40 y\left(t_{i+1}\right)+20 y\left(t_{i+2}\right)\right\} \\
S^{\prime \prime \prime}\left(t_{i-2}\right)+26 S^{\prime \prime \prime}\left(t_{i-1}\right)+66 S^{\prime \prime \prime}\left(t_{i}\right)+26 S^{\prime \prime \prime}\left(t_{i+1}\right)+S^{\prime \prime \prime}\left(t_{i+2}\right) \\
=\frac{1}{h^{3}}\left\{-60 y\left(t_{i-2}\right)+120 y\left(t_{i-1}\right)-120 y\left(t_{i+1}\right)+60 y\left(t_{i+2}\right)\right\}
\end{array}
$$




$$
\begin{aligned}
& S^{i v}\left(t_{i-2}\right)+26 S^{i v}\left(t_{i-1}\right)+66 S^{i v}\left(t_{i}\right)+26 S^{i v}\left(t_{i+1}\right)+S^{i v}\left(t_{i+2}\right) \\
& =\frac{1}{h^{4}}\left\{120 y\left(t_{i-2}\right)-480 y\left(t_{i-1}\right)+720 y\left(t_{i}\right)-480 y\left(t_{i+1}\right)+120 y\left(t_{i+2}\right)\right\}
\end{aligned}
$$

Using the operator notation $[6,16]$, the equations $(3.1)-(3.4)$ can we written as

$$
\begin{gathered}
S^{\prime}\left(t_{i}\right)=\frac{1}{h}\left(\frac{-5 E^{-2}-50 E^{-1}+50 E+5 E^{2}}{E^{-2}+26 E^{-1}+66 I+26 E+E^{2}}\right) y\left(t_{i}\right) \\
S^{\prime \prime}\left(t_{i}\right)=\frac{1}{h^{2}}\left(\frac{20 E^{-2}+40 E^{-1}-120 I+40 E+20 E^{2}}{E^{-2}+26 E^{-1}+66 I+26 E+E^{2}}\right) y\left(t_{i}\right) \\
S^{\prime \prime \prime}\left(t_{i}\right)=\frac{1}{h^{3}}\left(\frac{-60 E^{-2}+120 E^{-1}-120 E+60 E^{2}}{E^{-2}+26 E^{-1}+66 I+26 E+E^{2}}\right) y\left(t_{i}\right) \\
S^{i v}\left(t_{i}\right)=\frac{1}{h^{4}}\left(\frac{120 E^{-2}-480 E^{-1}+720 I-480 E+120 E^{2}}{E^{-2}+26 E^{-1}+66 I+26 E+E^{2}}\right) y\left(t_{i}\right)
\end{gathered}
$$

where the operators are defined as $E y\left(t_{i}\right)=y\left(t_{i}+h\right), D y\left(t_{i}\right)=y^{\prime}\left(t_{i}\right)$ and $\operatorname{Iy}\left(t_{i}\right)=$ $y\left(t_{i}\right)$. Let $E=e^{h D}$ and expand them in powers of $h D$, we get

$$
\begin{gathered}
S^{\prime}\left(t_{i}\right)=y^{\prime}\left(t_{i}\right)+\frac{1}{5040} h^{6} y^{7}\left(t_{i}\right)-\frac{1}{21600} h^{8} y^{9}\left(t_{i}\right)+\frac{1}{1036800} h^{10} y^{11}\left(t_{i}\right)+0\left(h^{11}\right) \\
S^{\prime \prime}\left(t_{i}\right)=y^{\prime \prime}\left(t_{i}\right)+\frac{1}{720} h^{4} y^{6}\left(t_{i}\right)-\frac{1}{3360} h^{6} y^{8}\left(t_{i}\right)+\frac{1}{86400} h^{8} y^{10}\left(t_{i}\right) \\
+\frac{221}{239500800} h^{10} y^{12}\left(t_{i}\right)+0\left(h^{11}\right) \\
S^{\prime \prime \prime}\left(t_{i}\right)=y^{\prime \prime \prime}\left(t_{i}\right)-\frac{1}{240} h^{4} y^{7}\left(t_{i}\right)+\frac{11}{30240} h^{6} y^{9}\left(t_{i}\right)-\frac{1}{28800} h^{8} y^{11}\left(t_{i}\right) \\
+\frac{37}{11404800} h^{10} y^{13}\left(t_{i}\right)+0\left(h^{11}\right) \\
S^{i v}\left(t_{i}\right)=y^{i v}\left(t_{i}\right)-\frac{1}{12} h^{2} y^{6}\left(t_{i}\right)+\frac{1}{240} h^{4} y^{8}\left(t_{i}\right)-\frac{1}{7560} h^{6} y^{10}\left(t_{i}\right) \\
-\frac{13}{907200} h^{8} y^{12}\left(t_{i}\right)+\frac{643}{159667200} h^{10} y^{14}\left(t_{i}\right)+0\left(h^{11}\right)
\end{gathered}
$$

We now define $e(t)=y(t)-S(t)$ and substitute equations (3.9)-(3.12) in the Taylor series expansion of $e\left(t_{i}+\theta h\right)$ we obtain

$$
\begin{gathered}
e\left(t_{i}+\theta h\right)=\left(\frac{\theta^{2}}{1440}-\frac{5 \theta^{4}}{1440}\right) h^{6} y^{6}\left(t_{i}\right)+\left(\frac{\theta}{5040}-\frac{\theta^{2}}{1440}\right) h^{7} y^{7}\left(t_{i}\right) \\
+\left(-\frac{\theta^{2}}{6720}+\frac{\theta^{4}}{5760}\right) h^{8} y^{8}\left(t_{i}\right)+0\left(h^{9}\right)
\end{gathered}
$$

where $a \leq \theta \leq b$. We abridge the above results in the following theorem:

Theorem 3.1. Let $y(t)$ be the exact solution and $S(t)$ be the numerical solution of the singularly perturbed fourth order boundary value problem (1.1) with the boundary conditions (1.2) for sufficiently small $h$ which further gives the truncation error of $O\left(h^{6}\right)$ and method of convergence of $O\left(h^{2}\right)$.

\section{Quasilinearization method}

Let us consider the boundary value problem

$$
\begin{gathered}
-\varepsilon y^{i v}(t)=F\left(t, y, y^{\prime \prime}, y^{\prime \prime \prime}\right), \quad t=[a, b], \\
y(a)=\eta_{1}, \quad y(b)=\eta_{2}, \quad y^{\prime \prime}(a)=\eta_{3}, \quad y^{\prime \prime}(b)=\eta_{4},
\end{gathered}
$$


where $F\left(t, y, y^{\prime \prime}, y^{\prime \prime \prime}\right)$ is a smooth function such that

$$
\left\{\begin{array}{l}
F_{y^{\prime \prime \prime}}\left(t, y, y^{\prime \prime}, y^{\prime \prime \prime}\right) \geq \alpha>0, \\
F_{y^{\prime \prime}}\left(t, y, y^{\prime \prime}, y^{\prime \prime \prime}\right) \geq \beta>0, \quad t \in[a, b] \\
0 \geq F_{y}\left(t, y, y^{\prime \prime}, y^{\prime \prime \prime}\right) \geq-\lambda, \quad \lambda>0 .
\end{array}\right.
$$

In order to obtain the numerical solution of the boundary value problem (4.1) and (4.2), Newton's method of quasilinearization $[1,4]$ is applied to generate the sequence of $\left\{y_{k}\right\}_{0}^{\infty}$ of successive approximations with a proper selection of initial guess $y_{0}$. We define $y_{k+1}$, for each fixed non-negative integer $k$, to be solution of the following linear problem:

$$
\begin{gathered}
-\varepsilon y_{k+1}^{i v}(t)-p_{k}(t) y_{k+1}^{\prime \prime \prime}(t)+q_{k}(t) y_{k+1}^{\prime \prime}(t)+r_{k}(t) y_{k+1}(t)=f_{k}(t), \quad t \in[a, b], \\
y_{k+1}(a)=\eta_{1}, \quad y_{k+1}(b)=\eta_{2}, \quad y_{k+1}^{\prime \prime}(a)=\eta_{3}, \quad y_{k+1}^{\prime \prime}(b)=\eta_{4}
\end{gathered}
$$

where

$$
\begin{aligned}
p_{k}(t)= & F_{y^{\prime \prime \prime}}\left(t, y_{k}, y_{k}^{\prime \prime}, y_{k}^{\prime \prime \prime}\right), q_{k}(t)=F_{y^{\prime \prime}}\left(t, y_{k}, y_{k}^{\prime \prime}, y_{k}^{\prime \prime \prime}\right) \\
r_{k}(t)= & F_{y}\left(t, y_{k}, y_{k}^{\prime \prime}, y_{k}^{\prime \prime \prime}\right), f_{k}(t)=F_{y}\left(t, y_{k}, y_{k}^{\prime \prime}, y_{k}^{\prime \prime \prime}\right) \\
& -y_{k} F_{y}\left(t, y_{k}, y_{k}^{\prime \prime}, y_{k}^{\prime \prime \prime}\right)-y_{k}^{\prime \prime} F_{y^{\prime \prime}}\left(t, y_{k}, y_{k}^{\prime \prime}, y_{k}^{\prime \prime \prime}\right) \\
& -y_{k}^{\prime \prime \prime} F_{y^{\prime \prime \prime}}\left(t, y_{k}, y_{k}^{\prime \prime}, y_{k}^{\prime \prime \prime}\right) .
\end{aligned}
$$

We make the following observations:

i) If the initial guess $y_{0}$ is sufficiently close to the solution $y(t)$ of (4.1) and (4.5), then the sequence $\left\{y_{k}\right\}_{0}^{\infty}$ converges to $y(x)$. One can see the proof given in [4]. From (4.3), it follows that, for each fixed $k$,

$$
\begin{aligned}
& p_{k}(t)=F_{y^{\prime \prime \prime}}\left(t, y, y^{\prime \prime}, y^{\prime \prime \prime}\right) \geq \alpha>0, \\
& q_{k}(t)=F_{y^{\prime \prime}}\left(t, y, y^{\prime \prime}, y^{\prime \prime \prime}\right) \geq \beta>0, \\
& 0 \geq r_{k}(t)=F_{y}\left(t, y, y^{\prime \prime}, y^{\prime \prime \prime}\right) \geq-\lambda, \quad \lambda>0 .
\end{aligned}
$$

ii) Problem (4.4) with the boundary conditions (4.5), for each fixed $k$, is a linear fourth order boundary value problem which is in the form of (1.1) and (1.2). Hence it can be solved by the method described in section 2 .

iii) The following convergence criterion is used to terminate the iteration:

$$
\left\|y_{k+1}\left(t_{i}\right)-y_{k}\left(t_{i}\right)\right\| \leq \varepsilon, \quad t_{i} \in[a, b], \quad k \geq 0 .
$$

\section{Numerical results}

In the present section, we have presented numerical results of the considered examples with the help of MATLAB software which verifies theoretical estimates.

When the exact solutions of the considered examples are available then the maximum absolute errors $E^{N}$ are evaluated using the following formula for the present method, which is given by

$$
E^{N}=\max _{t_{i} \in[a, b]}\left|y_{\varepsilon}^{N}\left(t_{i}\right)-S_{\varepsilon}^{N}\left(t_{i}\right)\right|,
$$


When the exact solutions of the considered examples are not available then the maximum absolute errors $E_{d}^{N}$ are evaluated using the double mesh principle for the present method, which is given by

$$
E_{d}^{N}=\max _{t_{i} \in[a, b]}\left|S_{\varepsilon}^{N}\left(t_{i}\right)-S_{\varepsilon}^{2 N}\left(t_{i}\right)\right|,
$$

The numerical order of convergence is computed using the following formula

$$
\operatorname{Ord}^{N}=\frac{\ln E^{N}-\ln E^{2 N}}{\ln 2} .
$$

The exact and approximate solutions are denoted by $y_{\varepsilon}^{N}$ and $S_{\varepsilon}^{N}$ respectively.

Example 5.1 Consider the following singular perturbation boundary value problem $[27]$ :

$$
\begin{gathered}
-\varepsilon y^{i v}(t)-4 y^{\prime \prime \prime}(t)=1, \quad t \in[0,1], \\
y(0)=1, \quad y(1)=1, \quad y^{\prime \prime}(0)=-1, \quad y^{\prime \prime}(1)=-1 .
\end{gathered}
$$

The exact solution of Example 5.1 is given by

\begin{tabular}{|c|c|c|c|c|c|c|c|c|c|c|}
\hline $\mathrm{N}$ & $\varepsilon=2^{-4}$ & Ord & $\varepsilon=2^{-5}$ & Ord & $\varepsilon=2^{-6}$ & Ord & $\varepsilon=2^{-7}$ & Ord & $\varepsilon=2^{-8}$ & Ord \\
\hline 64 & $9.5153 \mathrm{E}-06$ & 2.0442 & $1.1597 \mathrm{E}-05$ & 2.2186 & $9.3841 \mathrm{E}-06$ & 1.6594 & $7.6100 \mathrm{E}-06$ & 1.6904 & $1.0217 \mathrm{E}-05$ & 2.4174 \\
\hline 128 & $2.3070 \mathrm{E}-06$ & 2.0097 & $2.4916 \mathrm{E}-06$ & 2.0418 & $2.9708 \mathrm{E}-06$ & 2.2173 & $2.3579 \mathrm{E}-06$ & 1.6494 & $1.9126 \mathrm{E}-06$ & 1.6944 \\
\hline 256 & $5.7289 \mathrm{E}-07$ & 2.0010 & $6.0512 \mathrm{E}-07$ & 2.0095 & $6.3885 \mathrm{E}-07$ & 2.0421 & $7.5163 \mathrm{E}-07$ & 2.2142 & $5.9098 \mathrm{E}-07$ & 1.6435 \\
\hline 512 & $1.4312 \mathrm{E}-07$ & 2.1459 & $1.5029 \mathrm{E}-07$ & 1.4799 & $1.5512 \mathrm{E}-07$ & 2.0623 & $1.6198 \mathrm{E}-07$ & 2.0885 & $1.8916 \mathrm{E}-07$ & 2.2051 \\
\hline 1024 & $3.2339 \mathrm{E}-08$ & & $5.3882 \mathrm{E}-08$ & & $3.7140 \mathrm{E}-08$ & & $3.8086 \mathrm{E}-08$ & & $4.1024 \mathrm{E}-08$ & \\
\hline
\end{tabular}

$$
\begin{gathered}
y(t)=\frac{1}{192\left(1-e^{-\frac{4}{\varepsilon}}\right)}\left\{-3 \varepsilon^{2} e^{-\frac{4 t}{\varepsilon}}-72 t^{2}-8 t^{3}+80 t-3 t \varepsilon^{2}+192+3 \varepsilon^{2}\right. \\
\left.+e^{-\frac{4}{\varepsilon}}\left(-192+96 t^{2}+8 t^{3}-104 t+3 t \varepsilon^{2}\right)\right\} .
\end{gathered}
$$

Table 2. Maximum absolute errors and order of convergence of

Example 5.1 for different values of $\varepsilon$ and $N$.

Example 5.2. Consider the following singular perturbation boundary value problem $[7]$ :

$$
\begin{gathered}
-\varepsilon y^{i v}(t)+5 t y^{\prime \prime \prime}(t)+4 y^{\prime \prime}(t)+2 y(t)=0, \quad t \in[-1,1], \\
y(-1)=1, \quad y(1)=1, \quad y^{\prime \prime}(-1)=1, \quad y^{\prime \prime}(1)=1 .
\end{gathered}
$$

Table 3. Comparison of maximum absolute error and order of convergence of Example 5.2 for different values of $N$ and $\varepsilon=2^{-4}$.

\begin{tabular}{lccccc}
\hline$N$ & \multicolumn{2}{c}{ Geetha and Tamilselvan $[7]$} & & \multicolumn{2}{c}{ Present } \\
\cline { 2 - 3 } \cline { 5 - 6 } & $E_{d}^{N}$ & Ord & & $E_{d}^{N}$ & Ord \\
\hline 64 & $3.9249 \mathrm{E}-2$ & 0.9770 & & $4.4948 \mathrm{E}-04$ & 2.5794 \\
128 & $1.9940 \mathrm{E}-2$ & 0.9886 & & $7.5204 \mathrm{E}-05$ & 2.0864 \\
256 & $1.0049 \mathrm{E}-2$ & 0.9944 & & $1.7708 \mathrm{E}-05$ & 2.0177 \\
512 & $5.0440 \mathrm{E}-3$ & 0.9972 & & $4.3731 \mathrm{E}-06$ & 2.3071 \\
1024 & $2.5269 \mathrm{E}-3$ & & & $8.8366 \mathrm{E}-07$ & \\
\hline
\end{tabular}


Example 5.3. Consider the following singular perturbation boundary value problem $[7]$ :

$$
\begin{gathered}
-\varepsilon y^{i v}(t)+5 t y^{\prime \prime \prime}(t)+(4+t) y^{\prime \prime}(t)+\left(2+t^{2}\right) y(t)=-e^{t}+5, \quad t \in[-1,1], \\
y(-1)=1, \quad y(1)=1, \quad y^{\prime \prime}(-1)=2, \quad y^{\prime \prime}(1)=2 .
\end{gathered}
$$

Table 4. Comparison of maximum absolute error and order of convergence of Example 5.3 for different values of $N$ and $\varepsilon=2^{-4}$

\begin{tabular}{lccccc}
\hline \multirow{2}{*}{$N$} & \multicolumn{2}{c}{ Geetha and Tamilselvan $[7]$} & & \multicolumn{2}{c}{ Present } \\
\cline { 2 - 3 } \cline { 5 - 6 } & $E_{d}^{N}$ & Ord & & $E_{d}^{N}$ & Ord \\
\hline 64 & $3.3778 \mathrm{E}-2$ & 0.9823 & & $4.1824 \mathrm{E}-04$ & 2.5806 \\
128 & $1.7097 \mathrm{E}-2$ & 0.9913 & & $6.9920 \mathrm{E}-05$ & 2.0866 \\
256 & $8.6002 \mathrm{E}-3$ & 0.9957 & & $1.6462 \mathrm{E}-05$ & 2.0306 \\
512 & $4.3130 \mathrm{E}-3$ & 0.8693 & & $4.0291 \mathrm{E}-06$ & 2.1087 \\
1024 & $2.3610 \mathrm{E}-3$ & & & $9.3418 \mathrm{E}-07$ & \\
\hline
\end{tabular}

Example 5.4. Consider the following singular perturbation boundary value problem $[7]$ :

$$
\begin{gathered}
-\varepsilon y^{i v}(t)+5 t y^{\prime \prime \prime}(t)+(4+t) y^{\prime \prime}(t)+2 y^{2}(t)=0, \quad t \in[-1,1], \\
y(-1)=1, \quad y(1)=1, \quad y^{\prime \prime}(-1)=2, \quad y^{\prime \prime}(1)=2 .
\end{gathered}
$$

Table 5. Comparison of maximum absolute error and order of convergence of Example 5.4 for different values of $N$ and $\varepsilon=2^{-4}$

\begin{tabular}{lccccc}
\hline \multirow{2}{*}{$N$} & \multicolumn{2}{c}{ Geetha and Tamilselvan $[7]$} & & \multicolumn{2}{c}{ Present } \\
\cline { 2 - 3 } \cline { 5 - 6 } & $E_{d}^{N}$ & Ord & & $E_{d}^{N}$ & Ord \\
\hline 64 & $7.5762 \mathrm{E}-02$ & 0.9731 & & $1.1620 \mathrm{e}-03$ & 2.5795 \\
128 & $3.8593 \mathrm{E}-02$ & 0.9867 & & $1.9440 \mathrm{e}-04$ & 2.0863 \\
256 & $1.9475 \mathrm{E}-02$ & 0.9934 & & $4.5777 \mathrm{e}-05$ & 2.0215 \\
512 & $9.7821 \mathrm{E}-03$ & 0.9967 & & $1.1275 \mathrm{e}-05$ & 1.7449 \\
1024 & $4.9021 \mathrm{E}-03$ & - & & $3.3639 \mathrm{e}-06$ & - \\
\hline
\end{tabular}

\section{Conclusions}

In this article, we have used the quintic B-spline method for finding the approximate solution of fourth order linear and non-linear singular perturbation boundary value problems. We linearised the non-linear boundary value problem via quasilinearization method and solved the problem. It is a computationally proficient technique and the algorithm can easily be applied on a computer. The results obtained through this method are better than the existing method [7] with the same number of nodal points. 


\section{References}

[1] Bellman, R.E., Kalaba, R.E., Quasilinearization and Nonlinear Boundary Value Problems, American Elsevier, New York, 1965.

[2] Berger, A.E., Han, H., Kellogg, R.B., A priori estimates and analysis of a numerical method for a turning point problem, Mathematics of Computation, 42(1984), 465-492.

[3] Chen, S., Wang, Y., A rational spectral collocation method for third-order singularly perturbed problems, Journal of Computational and Applied Mathematics, 307(2016), 93-105.

[4] Doolan, E.P., Miller, J.J.H., Schildres, W.H.A., Uniform Numerical Method for Problems with Initial and Boundary Layers, Dublin, Boole Press, 1980.

[5] Farrell, P.A., Hagarty, A.F., Miller, J.J.H., O'Riordan, E., Shishkin, G.I., Robust Computational Techniques for Boundary Layers, Boca Raton, Chapman and Hall/CRC Press, 2000 .

[6] Fyfe, D.J., The use of the cubic spline in the solution of two point boundary value problems, Computing Journal, 12(1969), 188-192.

[7] Geetha, N., Tamilselvan, A., Parameter uniform numerical method for fourth order singularly perturbed turning point problems exhibiting boundary layers, Ain Shams Engineering Journal, http://dx.doi.org/10.1016/j.asej.2016.04.018, 2016.

[8] Geetha, N., Tamilselvan, A., Subburayan, V., Parameter uniform numerical method for third order singularly perturbed turning point problems exhibiting boundary layers, International Journal of Applied and Computational Mathematics, 2(2016), 349-364.

[9] Howes, F.A., The asymptotic solution of a class of third-order boundary value problem arising in the theory of thin film flow, SIAM Journal of Applied Mathematics, 43(1983), 993-1004.

[10] Kadalbajoo, M.K., Patidar, K.C., A survey of numerical techniques for solving singularly perturbed ordinary differential equations, Applied Mathematics and Computations, 130(2002), 457-510.

[11] Kadalbajoo, M.K., Yadaw, A.S., Kumar, D., Comparative study of singularly perturbed two-point BVPs via: Fitted-mesh finite difference method, B-spline collocation method and finite element method, Applied Mathematics and Computation, 204(2008), 713-725.

[12] Khan, A., Khan, I., Aziz, T., Sextic spline solution of a singularly perturbed boundaryvalue problems, Applied Mathematics and Computation, 181(2006), 432-439.

[13] Kumar, M., Singh, P., Mishra, H.K., A recent survey on computational techniques for solving singularly perturbed boundary value problems, International Journal of Computer Mathematics, 84(2007), 1439-1463.

[14] Lodhi, R.K., Mishra, H.K., Computational approach for fourth-order self-adjoint singularly perturbed boundary value problems via non-polynomial quintic spline, Iranian Journal of Science and Technology, Transactions A: Science, DOI 10.1007/s40995-0160116-6, 2016.

[15] Lodhi, R.K., Mishra, H.K., Solution of a class of fourth order singular singularly perturbed boundary value problems by quintic B-spline method, Journal of the Nigerian Mathematical Society, 35(2016), 257-265.

[16] Lucas, T.R., Error bounds for interpolating cubic splines under various end conditions, SIAM Journal Numerical Analysis, 11(1974), 569-584. 
[17] Miller, J.J.H., O'Riordan, E., Shishkin, G.I., Fitted numerical methods for singularly perturbed problems. Error estimates in the maximum norm for linear problems in one and two dimensions, Singapore: World Scientific Publishing Co. Pvt. Ltd., 2012.

[18] Mishra, H.K., Saini, S., Fourth order singularly perturbed boundary value problems via initial value techniques, Applied Mathematical Sciences, 8(2014), 619-632.

[19] O'Malley, R.E., Introduction to Singular Perturbations, New York, Academic Press, 1974.

[20] Pandit, S., Kumar, M., Haar wavelet approach for numerical solution of two parameters singularly perturbed boundary value problems, Applied Mathematics \& Information Sciences, 6(2014), 2965-2974.

[21] Rai, P., Sharma, K.K., Numerical analysis of singularly perturbed delay differential turning point problem, Applied Mathematics and Computation, 218(2011), 3486-3498.

[22] Rai, P., Sharma, K.K., Numerical study of singularly perturbed differential - difference equation arising in the modeling of neuronal variability, Computers \& Mathematics with Applications, 63(2012), 118-132.

[23] Roja, J.C., Tamilselvan,A., Shooting method for singularly perturbed fourth-order ordinary differential equations of reaction-diffusion type, International Journal of Computational Methods, http://dx.doi.org/10.1142/S0219876213500412, Vol. 10, 1350041 (21 pages), (2013).

[24] Roos, H.G., Stynes, M., Tobiska, L., Numerical Method for Singularly Perturbed Differential Equation Convection-Diffusion and Flow Problems, Springer, 2006.

[25] Sarakhsi, A.R., Ashrafi, S., Jahanshahi, M., Sarakhsi, M., Investigation of boundary layers in some singular perturbation problems including fourth order ordinary differential equations, World Applied Sciences Journal, 22(2013), 1695-1701.

[26] Sharma, K.K., Rai, P., Patidar, K.C., A review on singularly perturbed differential equations with turning points and interior layers, Applied Mathematics and Computation, 219(2013), 10575-10609.

[27] Shanthi, V., Ramanujam, N., A boundary value technique for boundary value problems for singularly perturbed fourth-order ordinary differential equations, Computers and Mathematics with Applications, 47(2004), 1673-1688.

[28] Valarmathi, S., Ramanujam, N., A computational method for solving third order singularly perturbed ordinary differential equations, Applied Mathematics and Computation, 129(2002), 345-373.

[29] Zhang, Y., Naidu, D. S., Cai, C., Zou, Y., Singular perturbations and time scales in control theories and applications: An overview 2002-2012, International Journal of Information and Systems Sciences, 9(2014), 1-35.

Ram Kishun Lodhi

Department of Mathematics, Jaypee University of Engineering \& Technology

AB Road Raghogarh, Guna-473226 (M.P.), India

e-mail: ramkishun.lodhi@gmail.com

Hradyesh Kumar Mishra

Corresponding author

Department of Mathematics, Jaypee University of Engineering \& Technology

AB Road Raghogarh, Guna-473226 (M.P.), India

e-mail: hk.mishra@juet.ac.in 